\title{
Spectral-Line Surveys at Millimeter and Submillimeter Wavelengths: Impact of Spectral Resolution
}

\section{M. Ziurys}

Depts. of Astronomy and Chemistry, and Arizona Radio Observatory, Steward Observatory, 933 N. Cherry Ave., University of Arizona, Tucson, AZ 85721

\begin{abstract}
Millimeter and submillimeter astronomy has achieved very high spectral resolution $\left(\lambda / \Delta \lambda \gtrsim 10^{6}-10^{7}\right)$ over the past 20 years. Broad-band spectralline surveys at these wavelengths have only taken partial advantage of these capabilities. Such surveys could be more effective with an increase in spectral resolution, accompanied by improvement in the stability and bandwidth of spectrometers and the employment of single-sideband detectors.
\end{abstract}

\section{Introduction}

The millimeter/sub-millimeter region of the electromagnetic spectrum, which covers the range $\approx 65-1000 \mathrm{GHz}$, is rich in molecular line emission. As a consequence, numerous broad-band spectral-line surveys have been conducted of molecular sources. The most frequently surveyed object is the Orion molecular cloud, observed in the $3 \mathrm{~mm}$ (Turner 1991), $2 \mathrm{~mm}$ (Ziurys \& McGonagle 1993), and $1 \mathrm{~mm}$ windows (Blake et al. 1986, Apponi et al. 2003), as well as in the sub-mm region (e.g., Araki et al. 2000). The Galactic center has also been studied (e.g., Nummelin et al. 2000), as well as a few other molecular clouds. Circumstellar envelopes, particularly that of the carbon-rich star IRC+10216, have additionally been investigated (e.g., Cernicharo et al. 2000).

These surveys have produced numerous scientific results, including the identification of new molecules such as $\mathrm{MgNC}$ and $\mathrm{C}_{5} \mathrm{H}$ (Cernicharo et al. 2000). Also, surveying different objects, or different positions within an object, has enabled an unbiased comparison of chemical variations (see Nummelin et al. 2000). Detailed studies of velocity components within a source, particularly in Orion, has also been accomplished. Futhermore, these surveys have demonstrated that there are numerous unidentified features, which have catalyzed new laboratory spectroscopy (e.g., Robinson, Apponi, \& Ziurys 1997).

\section{Limits of Spectral Resolution for Millimeter-wave Surveys}

Currently, very high spectral resolution is available at $\mathrm{mm} / \mathrm{sub}-\mathrm{mm}$ wavelengths, through the use of devices such as filter banks and autocorrelators. Radio telescopes are diffraction limited; hence, spectral resolution is solely determined by the spectrometer "backend." Resolutions as high as $24 \mathrm{kHz}$ have been successfully used, which correspond to $\lambda / \Delta \lambda \approx 10^{7}$. Therefore, very high 
spectral resolution has been available for the surveys. However, what is lacking in this regard is spectrometer baseline stability and bandwidth.

For example, most spectral surveys of Orion have been conducted with $1 \mathrm{MHz}$ resolution $\left(\approx 3 \mathrm{~km} \mathrm{~s}^{-1}\right.$ at $\left.3 \mathrm{~mm}\right)$. It would be very useful to survey Orion with higher resolution such as $100 \mathrm{kHz}$. This increase would enable the resolution of many velocity components in this object, as well as additional molecular splittings. An illustration of the improvement is shown in Figure 1, which displays a spectrum of the $\mathrm{J}=1 \rightarrow 0$ transition of a common interstellar molecule, $\mathrm{N}_{2} \mathrm{H}^{+}$, at $93 \mathrm{GHz}$, observed with $1 \mathrm{MHz}$ (left) and $100 \mathrm{kHz}$ (right) resolutions, respectively. In the $1 \mathrm{MHz}$ data, the transition appears virtually as a single feature. With $100 \mathrm{kHz}$ resolution, not only are the three components of the nitrogen hyperfine splitting resolved, but two velocity components in each feature become apparent as well. The two velocity components actually arise from separate clouds in Orion (Womack, Ziurys, \& Wyckoff 1991).
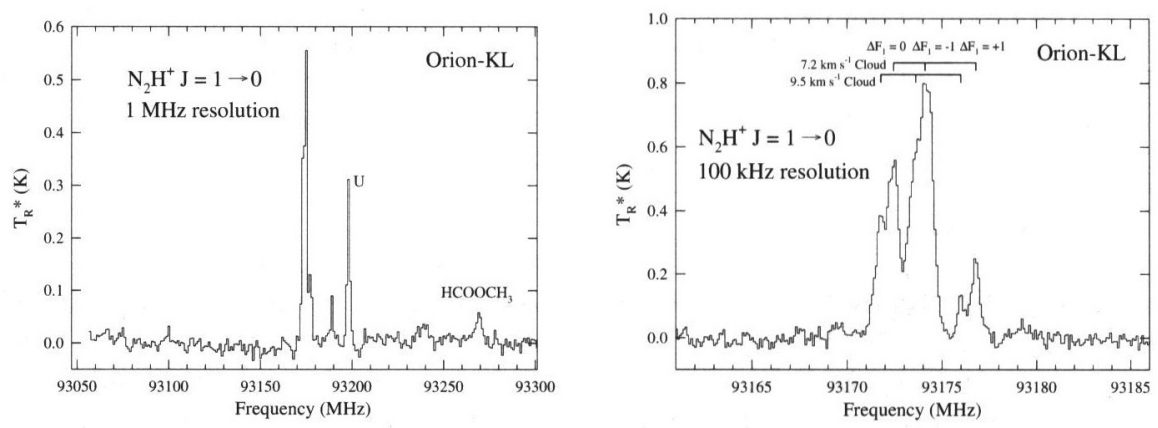

Figure 1. Spectra of $\mathrm{N}_{2} \mathrm{H}^{+}(\mathrm{J}=1 \rightarrow 0)$ at $93 \mathrm{GHz}$ observed towards Orion$\mathrm{KL}$ using the ARO $12 \mathrm{~m}$ telescope, showing the differences in line profiles for $1 \mathrm{MHz}$ (left) and $100 \mathrm{kHz}$ (right) resolution.

Data for spectral-line surveys need to be taken with reasonably large bandwidth $(0.5-1 \mathrm{GHz})$ for reasonable efficiency. Currently, it is difficult to make a broadband spectrometer with good baseline stability.

\section{The Double versus Single Sideband Problem}

Another difficulty for spectral surveys is a consequence of heterodyne detectors, which utilize local oscillators (LO) to mix down to an intermediate frequency (IF). Two frequency ranges are observed simultaneously with such systems, where $\nu_{S k y}=\nu_{L O} \pm \nu_{I F}$. Hence, the term "double sideband". Although at first glance this feature may seem an advantage, it leads to extreme spectral confusion at high sensitivity levels, and negates much of what is gained with increased resolution. Fortunately, rejection of the unwanted spectrum, or the "image" data, to generate a "single sideband" spectrum can be done, but not every telescope exercises this option. An illustration of the confusion created in double-sideband data is shown in Figure 2. This figure presents three spectra observed at $276.5 \mathrm{GHz}$ towards Orion-KL (Apponi et al. 2003). The bottom spectrum shows the single-sideband data, the middle one displays what would 
appear as the "image," and the top spectrum is the double sideband measurement. Distinct features clearly apparent in the SSB spectrum (e.g., the triplet near $276550 \mathrm{MHz}$ ), become either totally masked or blended in the DSB data. Clearly, surveys taken in DSB mode are losing information.

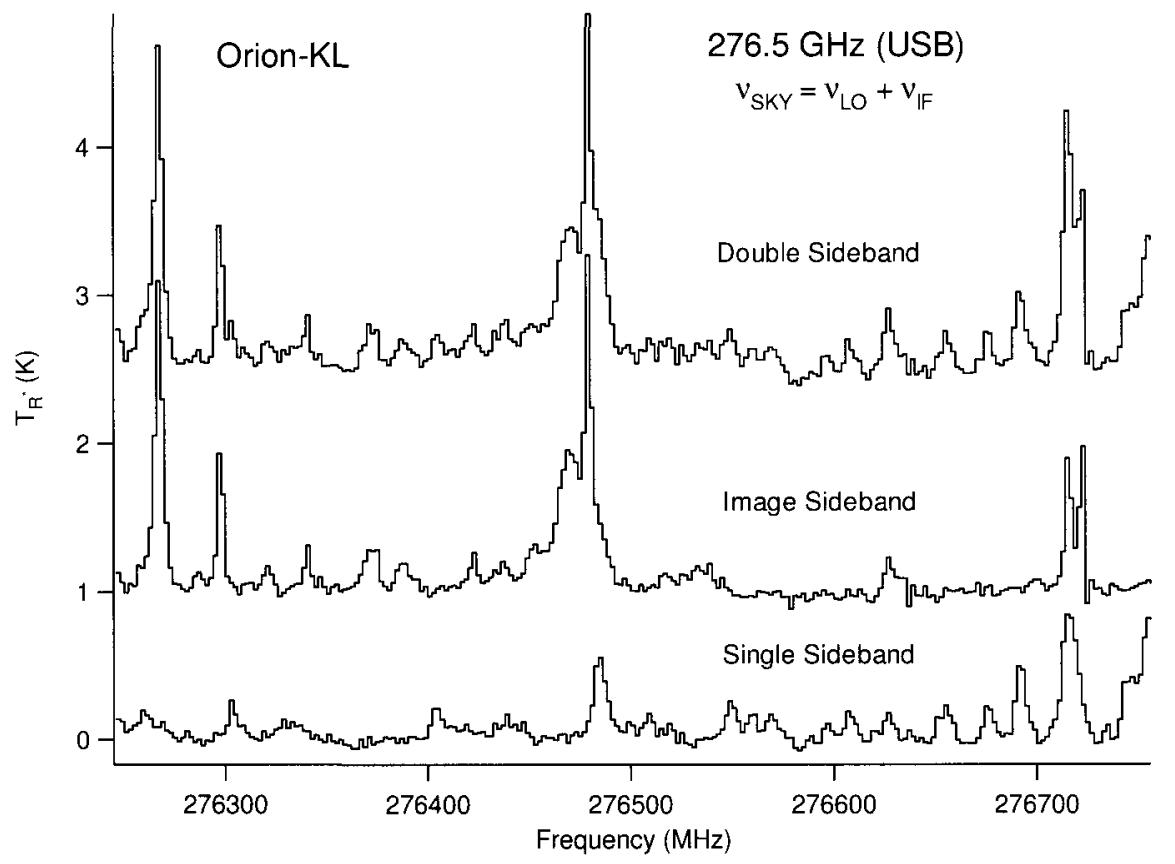

Figure 2. A comparison of spectra taken in single-sideband versus doublesideband modes.

\section{References}

Apponi, A. J., Halfen, D. T., \& Ziurys, L. M. 2003, in preparation

Araki, M., Ohishi, M., White, G. S., Higginbottom, N. S., Greves, J. S. 2000, in Atomic and Molecular Data for Astrophysics, IAU General Assembly, JD 1

Blake, G. A., Masson, C., Phillips, T. G., \& Sutton, E. 1986, ApJS, 60, 357

Cernicharo, J., Guélin, M., \& Kahane, C. 2000, A\&AS, 142, 181

Nummelin, A., et al. 2000, ApJS, 128, 213

Robinson, J., Apponi, A. J., \& Ziurys, L. M. 1997, Chem. Phys. Lett., 278, 1

Turner, B. E. 1991, ApJS, 76, 617

Womack, M. P., Ziurys, L. M., \& Wyckoff, S. 1991, ApJL, 370, L99

Ziurys, L. M. \& McGonagle, D. 1993, ApJS, 89, 155 